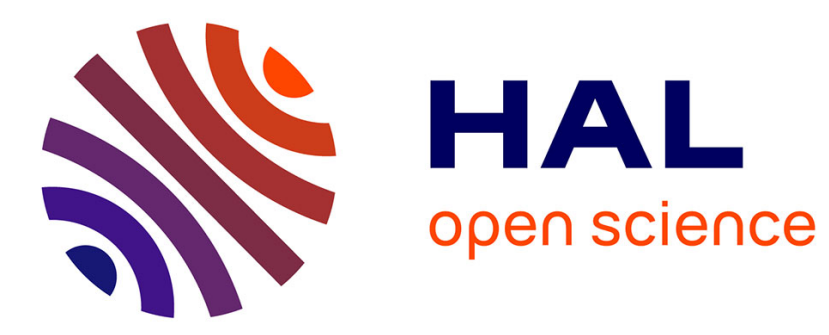

\title{
Origine des centres recombinants aux joints de grains de bicristaux de silicium $\Sigma=13$
}

\author{
H. Amanrich, M. Pasquinelli, S. Martinuzzi
}

\section{To cite this version:}

H. Amanrich, M. Pasquinelli, S. Martinuzzi. Origine des centres recombinants aux joints de grains de bicristaux de silicium $\Sigma=13$. Revue de Physique Appliquée, 1990, 25 (11), pp.1121-1128. 10.1051/rphysap:0199000250110112100 . jpa-00246282

\section{HAL Id: jpa-00246282 https://hal.science/jpa-00246282}

Submitted on 1 Jan 1990

HAL is a multi-disciplinary open access archive for the deposit and dissemination of scientific research documents, whether they are published or not. The documents may come from teaching and research institutions in France or abroad, or from public or private research centers.
L'archive ouverte pluridisciplinaire HAL, est destinée au dépôt et à la diffusion de documents scientifiques de niveau recherche, publiés ou non, émanant des établissements d'enseignement et de recherche français ou étrangers, des laboratoires publics ou privés. 


\title{
Origine des centres recombinants aux joints de grains de bicristaux de silicium $\Sigma=13$
}

\author{
H. Amanrich, M. Pasquinelli et S. Martinuzzi \\ Laboratoire de Photoélectricité des Semi-conducteurs, Université d'Aix-Marseille III, Centre de St. Jérôme, \\ F - 13397 Marseille Cedex 13, France
}

(Reçu le 5 février 1990, révisé le 11 avril 1990, accepté le 29 juin 1990)

\begin{abstract}
Résumé. - Pour vérifier que la ségrégation d'oxygène est bien à l'origine de l'activité recombinante des joints de grains de bicristaux « $C Z$ », de configuration $\Sigma=13$ et de type $P$, des échantillons ont été étudiés bruts de fabrication, après recuit, après diffusion d'or ainsi qu'après recuit et diffusion d'or. Les techniques expérimentales regroupent les balayages LBIC, le contraste EBIC, ainsi que la DLTS utilisant la région de charge d'espace du joint de grains comme structure de collecte. Les résultats montrent que l'activité recombinante des joints ne devient notable qu'après un recuit à $880{ }^{\circ} \mathrm{C}$ pendant plusieurs dizaines d'heures. Une diffusion d'or à $700{ }^{\circ} \mathrm{C}$ ne produit pas d'autre effet que celui dû au seul recuit. Une diffusion d'or à $800{ }^{\circ} \mathrm{C}$ pendant 3 heures dans un bicristal préalablement recuit à $880{ }^{\circ} \mathrm{C}$ pendant 30 heures réduit les recombinaisons aux joints et dans les grains. L'activation des joints de grains de type $\Sigma=13$ est donc un phénomène lent, tandis que la présence d'impuretés métalliques diffusant rapidement dans le silicium est soit sans effet, soit passivante. L'activation des joints doit donc résulter de la capture de diffuseurs relativement lents. Par suite de son abondance dans les cristaux « $\mathrm{CZ}$ » et des précipités qu'il peut former aux joints, l'oxygène dissous dans les grains apparaît bien après ségrégation comme le principal responsable de l'activation de ces défauts.
\end{abstract}

\begin{abstract}
In order to verify that the recombination strength of minority carriers within grain boundaries (GBs) in p type silicon " $\mathrm{CZ}$ » bicrystals $(\Sigma=13)$ results of the segregation of dissolved oxygen we have investigated, as grown, annealed, gold diffused and also annealed then gold diffused samples. The investigations are made by means of LBIC scan maps, EBIC contrast and DLTS through the space charge region of the grain boundary. Annealing are carried out in argon. The results show that the recombination strength becomes noticeable after annealing at $880{ }^{\circ} \mathrm{C}$ for several tens of hours only, but is insensitive to gold in-diffusion at $700{ }^{\circ} \mathrm{C}$. In a bicrystal annealed at $880{ }^{\circ} \mathrm{C}$ for 30 hours, gold diffusion at $800{ }^{\circ} \mathrm{C}$ for 3 hours reduces the recombination strength at GBs and in the grains. It is clear that the activation of $\Sigma=13 \mathrm{GBs}$ results of a slow process. As the density of dissolved oxygen is very high in " $\mathrm{CZ}$ " crystals and as oxygen is able to precipitate at the annealing temperatures used in the present work, it is concluded that the segregation of oxygen is the most likely mechanism to explain the activation of GBs.
\end{abstract}

\section{- ntro uction.}

Les joints de grains sont les défauts cristallographiques étendus dominants dans la plupart des semiconducteurs polycristallins. Par la barrière de potentiel qu'ils opposent au déplacement des porteurs majoritaires, ils diminuent la mobilité de ces porteurs. Par les recombinaisons de paires électronstrous qu'ils peuvent développer, ils affectent directement la durée de vie des porteurs minoritaires dans leur voisinage immédiat. Il est admis que la barrière de potentiel est due au piègeage des porteurs dans le plan du joint de grains. Ces pièges profonds peuvent être générés par la présence de liaisons pendantes, mais ces liaisons ont peu de chance de se trouver en densité importante par suite de la reconstruction du réseau. Aussi est-ce la ségrégation d'impuretés par le joint de grains, impuretés prélevées le plus souvent dans les grains, qui crée les états d'interface localisés dans la bande interdite du joint de grains. Il est en effet apparu que l'activité recombinante superficielle et la hauteur de barrière $E_{B}$ ne dépend pas (ou très peu) du type de joint de 
grains, qu'elle est tributaire de l'histoire thermique du matériau et qu'elle est affectée par la présence d'impuretés recombinantes résiduelles ou introduites volontairement dans le cristal. Dans le silicium, il a été constaté qu'en plus des diffuseurs rapides, l'oxygène et même le carbone, toujours présents en quantité importante dans ce matériau, jouent un rôle majeur dans l'activation des joints de grains de bicristaux durant les traitements thermiques [1-6].

Toutefois, cette interprétation est contestée par d'autres auteurs qui remettent en cause l'importance de l'oxygène en se fondant sur la faible valeur du coefficient de diffusion de cet élément, et qui privilégient la ségrégation d'impuretés métalliques comme le cuivre qu'ils détectent par microanalyse aux joints de grains de bicristaux obtenus par tirage CZOCHRALSKI ( $\mathrm{CZ} »)$ [7].

Pourtant des recuits à des températures élevées supérieures à $800^{\circ} \mathrm{C}$ pendant plusieurs dizaines d'heures sont nécessaires pour augmenter sensiblement les recombinaisons aux joints de grains de bicristaux $[3,8]$.

Des températures aussi élevées et des durées de recuit aussi longues ne sont pas nécessaires pour déplacer les diffuseurs rapides des grains jusqu'au joint où ils peuvent être capturés. Même si ces impuretés métalliques se trouvent en substitution elles peuvent par le mécanisme de "kick-out» passer en position interstitielle, puisque une sursaturation en self interstitiels se développe dans les bicristaux « $\mathbf{C Z}$ » au cours des recuits par suite de la formation de précipités [3].

Aussi sans exclure a priori, l'intervention d'atomes métalliques dans le mécanisme d'activation électrique des joints de grains de type $\Sigma=13$, il nous paraît légitime d'admettre que cette activation est due soit à la transformation lente du joint par capture d'un diffuseur lent comme l'oxygène dissous dans les grains, soit à la capture précédente suivie de celle d'éléments métalliques.

Dans ce travail, nous essayons d'élucider ce problème en montrant que, dans les bicristaux de type $\Sigma=13$ un recuit préalable sous flux d'argon modifie à la fois l'activité électrique du joint de grains et l'influence sur cette activité d'un diffuseur rapide, comme l'or, introduit dans les grains loin du joint. Les conditions expérimentales sont choisies de sorte que cet élément puisse se trouver en position interstitielle dans la plus grande part du bicristal, sauf au joint de grains où il serait capturé. Cette réaction de l'or avec les défauts des cristaux de silicium est un phénomène bien connu, qui peut même être utilisé pour révéler leur présence et comprendre leurs propriétés [9].

\section{Techniques expérimentales.}

Des échantillons de $10 \times 2 \times 1 \mathrm{~mm}^{3}$ ont été découpés dans des bicristaux de silicium de type $P$, de résisti- vité $20 \Omega . \mathrm{cm}$, où le joint de grains traverse l'échantillon [10]. Ces joints résultent de la rotation d'un angle de $22,67^{\circ}$ autour de l'axe [001], le plan d'interface a pour indice (510). Les axes communs sont orientés [001] et [510] [11]. La concentration en oxygène interstitiel est supérieure à $10^{18} \mathrm{~cm}^{-3}$. Ces échantillons peuvent être étudiés bruts de fabrication ou après avoir subi divers recuits, une diffusion d'or ou encore un recuit et une diffusion d'or. Les traitements thermiques sont effectués dans un four à tube ouvert dans un flux d'argon. Pour la diffusion d'or, le métal est déposé par pulvérisation cathodique de part et d'autre du joint de grains à moins de $1 \mathrm{~mm}$ du joint, puis l'ensemble est recuit sous flux d'argon à 700 et $800^{\circ} \mathrm{C}$. Ces températures sont choisies de façon que les atomes d'or en position interstitielle diffusent dans tout le matériau $\left(D_{\text {Aui }} \simeq 10^{-6} \mathrm{~cm}^{2} \mathrm{~s}^{-1}\right)$ et parviennent au joint où ils peuvent être capturés, alors qu'à $800{ }^{\circ} \mathrm{C}$ la diffusion en position substitutionnelle de l'or est encore limitée à quelques micromètres $\left(D_{\text {Aus }}=\right.$ $\left.10^{-12} \mathrm{~cm}^{2} . \mathrm{s}^{-1}\right)[12,13]$.

Seul l'or en position substitutionnelle donne des centres de recombinaison dans le silicium se traduisant par des niveaux profonds dans la bande interdite, tandis que les atomes d'or en position interstitielle diffusés dans les grains n'ont aucune activité recombinante $[12,13]$. En revanche, ceux capturés par le joint de grains pourraient éventuellement en avoir une et ainsi modifier l'activité électrique du joint. L'introduction d'atomes d'or dans les échantillons peut être faite avant tout recuit ou après des traitements thermiques préalables.

Après chaque traitement thermique, les échantillons sont très rapidement refroidis $\left(200{ }^{\circ} \mathrm{C}\right.$ par minute) puis ils font l'objet d'une attaque chimique afin d'éliminer les couches d'oxyde avant les dépôts métalliques nécessaires à la réalisation des contacts ohmiques et redresseurs.

De même, après les diffusions d'or, les échantillons sont polis mécaniquement puis chimiquement avant le dépôt des contacts. Les contacts ohmiques sont faits par dépôt d'une couche d'aluminium de $3000 \AA$ d'épaisseur, suivi par un recuit à $450{ }^{\circ} \mathrm{C}$ pendant une heure sous flux d'argon. Ces contacts sont placés de part et d'autre du joint (Fig. 1a). Les contacts redresseurs semi-transparents sont obtenus par condensation d'une couche d'aluminium de $100 \AA$ d'épaisseur recouvrant le joint de grains et le contact ohmique est alors réalisé à froid avec un amalgame In-Ga (Fig. 1b).

L'activité recombinante des joints de grains est évaluée par deux méthodes indépendantes :

- La D.L.T.S. (Deep Level Transient Spectroscopy) [14], méthode globale utilise ici la région de charge d'espace liée à la barrière de potentiel du joint. Le joint constitue donc lui-même la structure de redressement nécessaire à ces mesures [15-16]. 

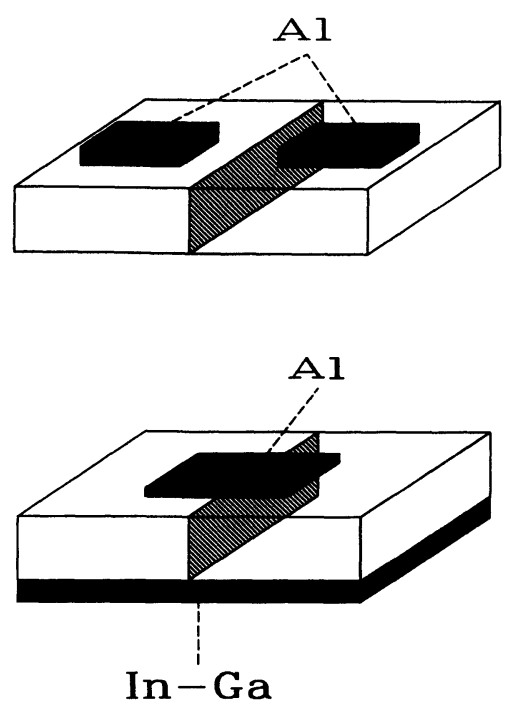

Fig. 1. - Schémas des structures d'étude: (a) contacts ohmiques de part et d'autre du joint de grains ; (b) contact semitransparent et redresseur pour l'étude des recombinaisons au joint.

[Sketch of the investigation structures : (a) ohmic contacts used for D.L.T.S.; (b) semitransparent and rectifying contact used for the study of the recombinations within the grain boundary.]

Cette technique conduit aux valeurs de $E_{\mathrm{T}}, N_{\mathrm{T}}$ et $\sigma_{\mathrm{T}}$, qui sont respectivement l'énergie au-dessus de la bande de valence $\left(E_{\mathrm{v}}+E_{\mathrm{T}}\right)$, la densité et la section efficace de capture des centres pièges de porteurs majoritaires, les calculs détaillés ont déjà été publiés $[14,17]$. La valeur $N_{\mathrm{T}}$, donnée par cette méthode, concerne le volume délimité par l'aire du joint et la largeur $W$ de la région de charge d'espace. Si le nombre de centres pièges liés au joint est très grand devant celui contenu dans la région de charge d'espace, la mesure nous renseigne essentiellement sur ce qui se produit au joint de grains avec une bonne approximation. Si cette condition n'est pas bien vérifiée, $N_{\mathrm{T}}$ et $\sigma_{\mathrm{T}}$ ne pourront être donnés qu'à titre indicatif. Nous admettrons dans la suite que $N_{\mathrm{T}}$ est aussi la densité de centres recombinants pour les minoritaires, puisque la formation de la barrière de potentiel liée au joint, résulte du piégeage des majoritaires et que ce sont les centres ainsi chargés qui capturent les minoritaires et les recombinent.

- Les balayages L.B.I.C. (Light Beam Induced Current) dans le proche infra-rouge $(\lambda=940 \mathrm{~nm})$ avec un spot de $10 \mu \mathrm{m}$ de diamètre permettent de dessiner une image photoélectrique tridimensionnelle du bicristal, d'évaluer localement la vitesse de recombinaison interfaciale $S$ le long du joint et de mesurer localement la longueur de diffusion $L_{\mathrm{n}}$ des électrons dans les grains et près du joint [18]. Nous pouvons ainsi vérifier que les variations de $S$ sont corrélées avec la présence de niveaux d'énergie profonds aux joints de grains, donc avec la hauteur de la barrière de potentiel associée à ces défauts [19].

Le contraste E.B.I.C. est également utilisé pour observer l'existence et surtout l'homogénéité de l'activité recombinante des joints de grains après les divers traitements appliqués aux échantillons. La séquence des différentes opérations et des mesures est donnée par la figure 2.

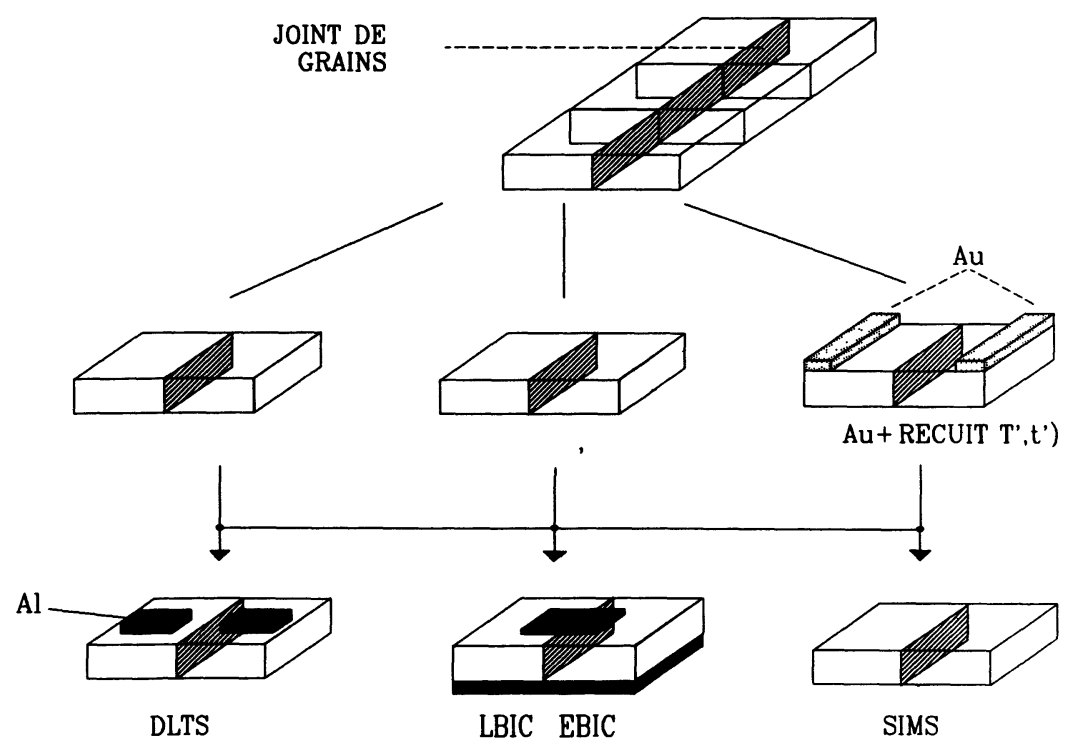

Fig. 2. - Séquences expérimentales d'étude des joints de grains bruts, recuits, recuits et soumis à une diffusion d'or.

[Experimental steps for the study of the grain boundary in as grown bicrystals, annealed bicrystals, annealed and gold diffused bicrystals.] 
Tableau I. - Resultats obtenus par LBIC et DLTS avec des bicristaux bruts, après recuits, après diffusion d'or ainsi qu'après recuit et diffusion d'or.

[Results given by LBIC and DLTS techniques for as grown, annealed, gold diffused and annealed and gold diffused bicrystals.]

\begin{tabular}{|c|c|c|c|c|c|c|}
\hline Traitements & $\begin{array}{r}L \\
\text { grain }\end{array}$ & joint & $\underset{\left(\mathrm{cm} \cdot \mathrm{s}^{-1}\right)}{S}$ & $\begin{array}{c}E_{\mathrm{T}} \\
(\mathrm{eV})\end{array}$ & $\begin{array}{c}N_{\mathrm{T}} \\
\left(\mathrm{cm}^{-3}\right)\end{array}$ & $\underset{\left(\mathrm{cm}^{2}\right)}{\mathrm{T}}$ \\
\hline Brut & 50 & 45 & $2 \times 10^{3}$ & - & - & - \\
\hline $700^{\circ} \mathrm{C} 2 \mathrm{~h}$ & 70 & 44 & $3 \times 10^{3}$ & - & - & - \\
\hline $880{ }^{\circ} \mathrm{C} 30 \mathrm{~h}$ & 20 & 7 & $8 \times 10^{3}$ & \multicolumn{3}{|c|}{ Pas analysable } \\
\hline $880^{\circ} \mathrm{C} 48 \mathrm{~h}$ & 14 & 7 & $1 \times 10^{4}$ & 0.40 & $3 \times 10^{16}$ & $10^{-18}$ \\
\hline $\mathrm{Au}+700^{\circ} \mathrm{C} 2 \mathrm{~h}$ & 70 & 44 & $3 \times 10^{3}$ & - & - & - \\
\hline $\mathrm{Au}+800{ }^{\circ} \mathrm{C} 3 \mathrm{~h}$ & 66 & 46 & $2 \times 10^{3}$ & - & - & - \\
\hline $880^{\circ} \mathrm{C}+30 \mathrm{~h}+\mathrm{Au}+800^{\circ} \mathrm{C} 3 \mathrm{~h}$ & 40 & 15 & $8 \times 10^{-3}$ & - & - & - \\
\hline $880{ }^{\circ} \mathrm{C}+48 \mathrm{~h}+\mathrm{Au}+800{ }^{\circ} \mathrm{C} 3 \mathrm{~h}$ & 40 & 25 & $8 \times 10^{3}$ & 0.38 & $5 \times 10^{14}$ & $8 \times 10^{13}$ \\
\hline
\end{tabular}

\section{Résultats.}

Le tableau I condense l'ensemble des résultats obtenus. Le joint du bicristal brut est très faiblement actif: il n'y a pas de capacité mesurable et les caractéristiques courant-tension sont presque linéaires. Une faible atténuation de photocourant et de longueur de diffusion est observable dans les balayages L.B.I.C. (Fig. 4a) et il est possible d'en déduire une valeur de $S$ qui serait au plus égale à $10^{3} \mathrm{~cm} . \mathrm{s}^{-1}$. Ces résultats sont en particulier en accord avec ceux de Battistella et al. [3], et de Stuzler et Queisser [4]. Des traitements thermiques ne modifient pas sensiblement l'activité du joint tant que la température reste inférieure à $800^{\circ} \mathrm{C}$ et la durée inférieure à une dizaine d'heures. Toutefois $S$ a tendance à augmenter et $L_{\mathrm{n}}$ près du joint diminue au cours de ces traitements. Ce n'est que pour des températures plus élevées et pour des durées plus longues que des modifications appréciables sont observées. Après 30 heures à $880^{\circ} \mathrm{C}$, une capacité électrique est mesurable, les caractéristiques courant-tension sont dissymétriques, les longueurs de diffusion dans les grains et surtout près du joint sont fortement diminuées et $S$ atteint $8 \times 10^{3} \mathrm{~cm} \cdot \mathrm{s}^{-1}$. Un spectre D.L.T.S. est observable, mais il ne peut pas être exploité par suite de la largeur exagérée des pics et du bruitage des signaux.

Si la durée du traitement à $880{ }^{\circ} \mathrm{C}$ est prolongée et passe à 48 heures, une évolution radicale se produit : l'activité recombinante est nettement accrue, $S$ atteint $1,4 \times 10^{4} \mathrm{~cm} . \mathrm{s}^{-1}$, et les spectres de D.L.T.S. deviennent exploitables (Fig. 5a). La variation de l'amplitude des pics avec le taux d'émission suggère l'existence d'une bande de niveaux pièges autour de $E_{\mathrm{T}}=0,4 \mathrm{eV}$, la densité $N_{\mathrm{T}}$ vaut $10^{16} \mathrm{~cm}^{-3}$ et la section efficace de capture $\sigma_{\mathrm{T}}$ serait de l'ordre de $10^{-8} \mathrm{~cm}^{2}$. Les longueurs de diffusion s'effondrent dans les grains comme aux joints comme le montre le balayage L.B.I.C. de la figure 4 b. Ces évolutions sont confirmées par le contraste et les balayages

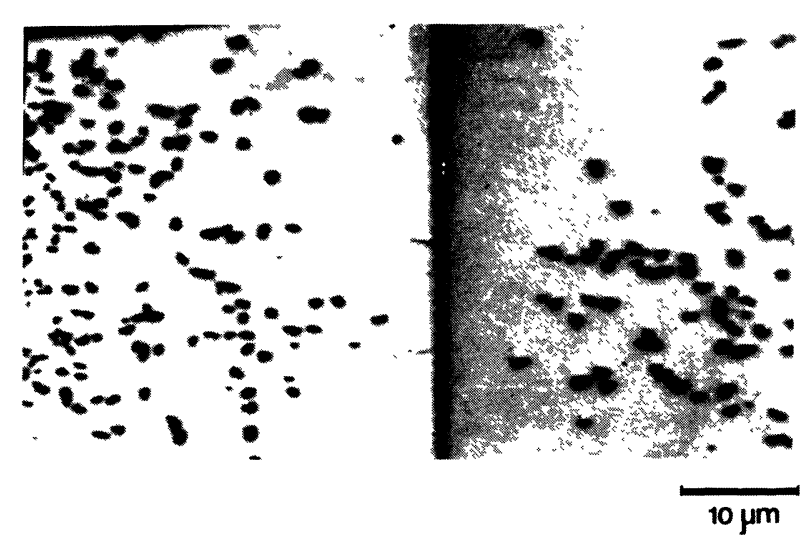

Fig. 3. - Contraste EBIC au voisinage d'un joint de grains après un recuit à $880{ }^{\circ} \mathrm{C}$ pendant $48 \mathrm{~h}$. Le joint présente un contraste ponctué et autour du joint apparaît une zone exempte de groupes de centres recombinants.

[EBIC contrast within a grain boundary (G.B.) after annealing at $880{ }^{\circ} \mathrm{C}$ for $48 \mathrm{~h}$. The G.B. exhibits a dotted contrast and around the G.B. a denuded zone appears in which recombining centre groups are not revealed.] 
E.B.I.C. Le contraste qui est peu marqué et homogène dans les bicristaux bruts ou insuffisamment recuits, se renforce et devient ponctué après un traitement de 30 heures à $880{ }^{\circ} \mathrm{C}$, comme le montre la figure 3. De plus, après 48 heures à $880{ }^{\circ} \mathrm{C} \mathrm{le}$ contraste ponctué du joint devient plus intense et de part et d'autre du joint une région "dénudée " large d'environ $30 \mu \mathrm{m}$ s'est formée dans laquelle il n'y a pas de recombinaisons. Au delà de cette région réapparaît dans les grains un contraste ponctué dû à des groupes de centres recombinants. Ces résultats sont en accord avec ceux de Battistella et al. [3], d'Ilhal et al. [20], ainsi que de Pollock et al. [1]. La largeur de cette région dénudée augmente avec la durée du recuit à $880^{\circ} \mathrm{C}$, ce qui montre qu'elle ne s'est pas entièrement développée lors de la croissance du bicristal.

Remarque : les bicristaux recuits à $880{ }^{\circ} \mathrm{C}$ présentent des fautes d'empilement, et lorsqu'ils sortent du four leur surface est oxydée par l'exodiffusion de l'oxygène dissous. (Des monocristaux préparés par fusion de zone (F. Z.) placés à côté des bicristaux et donc soumis au même traitement thermique ont eux conservé leur aspect initial).

Si avant tout recuit, de l'or est diffusé à $700{ }^{\circ} \mathrm{C}$ pendant 2 heures ou même à $800{ }^{\circ} \mathrm{C}$ pendant 3 heures, les propriétés du joint changent peu et se confondent avec celles observées après un recuit seul dans les mêmes conditions de température et de durée. La présence d'or en solution interstitielle dans le bicristal et en particulier près du joint, n'a aucun effet mesurable sur l'activité électrique de ce défaut, bien que les solubilités limites de l'or à $800{ }^{\circ} \mathrm{C}$ soient d'environ $10^{15} \mathrm{~cm}^{-3}$ pour $\mathrm{Au}_{\mathrm{s}}$ et $10^{14} \mathrm{~cm}^{-3}$ pour $\mathrm{Au}_{\mathrm{i}}$ (concentrations qui sont décelables par la D.L.T.S.). Le balayage L.B.I.C. de la figure $4 \mathrm{c}$ illustre ce comportement.

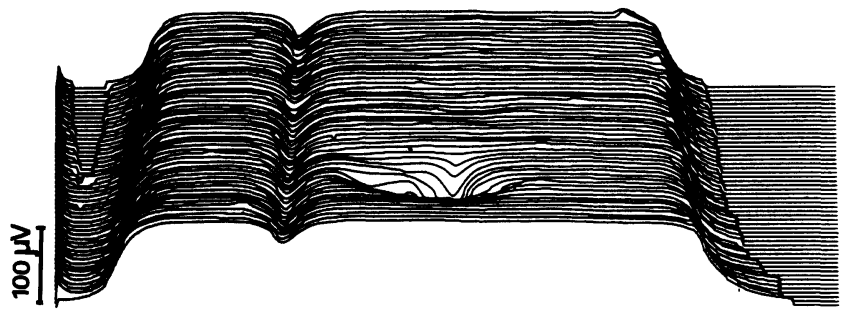

$\overrightarrow{500 \mu \mathrm{m}}$

a)

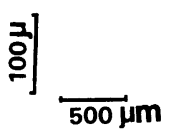

c)

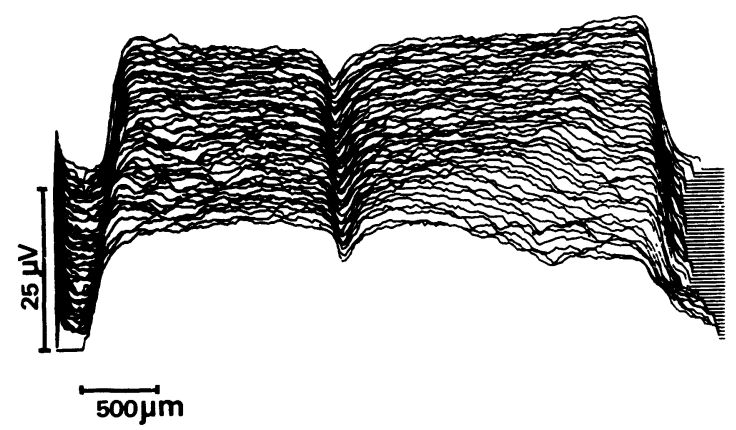

b)

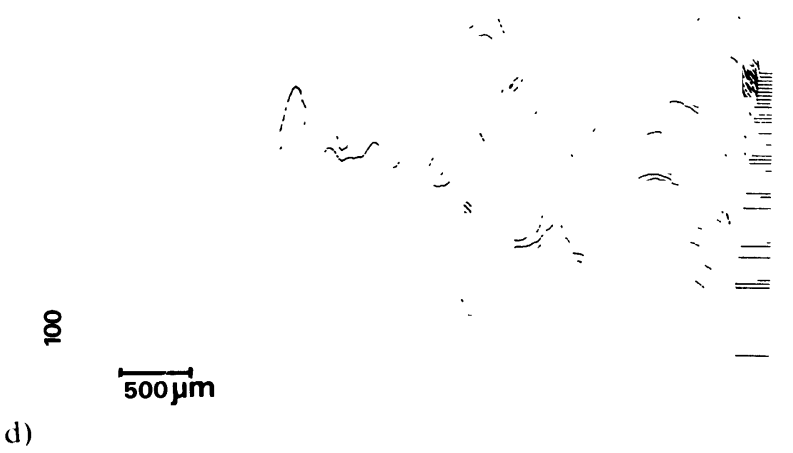

Fig. 4. - Images LBIC à $\lambda=940 \mathrm{~nm}$ d'un joint de grains brut (a) et après différents traitements : (b) recuit à $880{ }^{\circ} \mathrm{C}$ pendant $48 \mathrm{~h} ;$; (c) après diffusion d'or à $600{ }^{\circ} \mathrm{C}$ pendant $2 \mathrm{~h}$; (d) après un recuit à $880{ }^{\circ} \mathrm{C}$ pendant $48 \mathrm{~h}$ et une diffusion d'or à $800{ }^{\circ} \mathrm{C}$ pendant $3 \mathrm{~h}$.

[LBIC scan map at $\lambda=940 \mathrm{~nm}$ within a grain boundary (a) : as grown; (b) annealed at $880{ }^{\circ} \mathrm{C}$ for $48 \mathrm{~h}$; (c) after gold diffusion at $600{ }^{\circ} \mathrm{C}$ for $2 \mathrm{~h}$; (d) after annealing at $880{ }^{\circ} \mathrm{C}$ for $48 \mathrm{~h}$ and gold diffusion at $800{ }^{\circ} \mathrm{C}$ for $3 \mathrm{~h}$. 


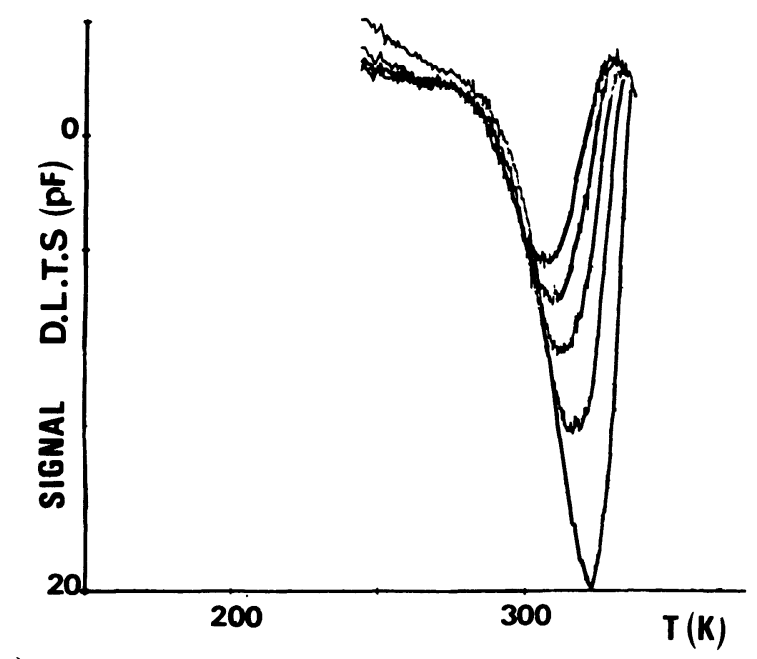

a)

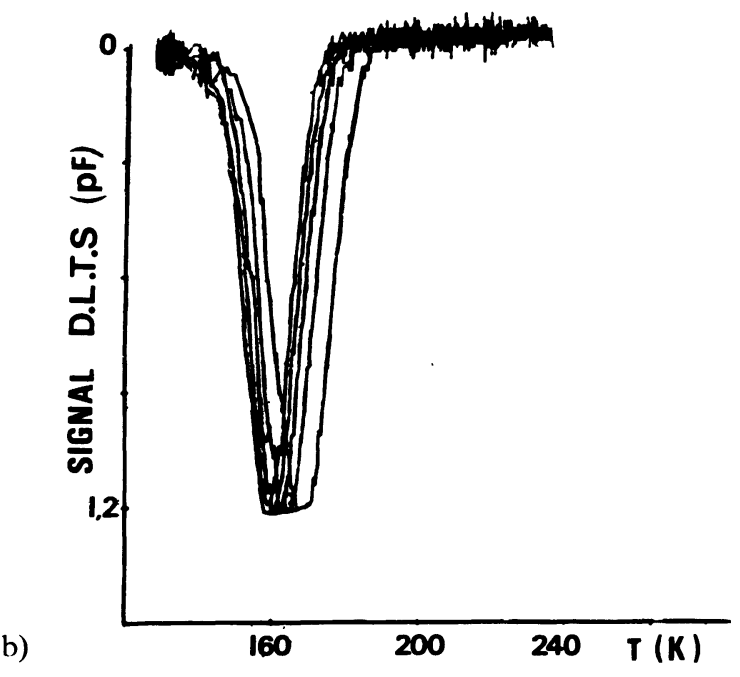

Fig. 5. - Spectres DLTS obtenus (a) après recuit à $8800^{\circ} \mathrm{C}$ pendant $\quad 48 \mathrm{~h} \quad\left(E_{\mathrm{T}}=0,4 \mathrm{eV} ; \quad N_{\mathrm{T}}=10^{16} \mathrm{~cm}^{-3}\right.$; $\sigma_{\mathrm{T}}=10^{-18} \mathrm{~cm}^{2}$ ); (b) après le recuit précédent et diffusion d'or à $800{ }^{\circ} \mathrm{C} \quad$ pendant $3 \mathrm{~h} \quad\left(E_{\mathrm{T}}=0,38 \mathrm{eV}\right.$; $N_{\mathrm{T}}=5.10^{14} \mathrm{~cm}^{-3} ; \quad \sigma_{\mathrm{T}}=10^{-12} \mathrm{~cm}^{2}$ ). Pour les deux spectres (a) et (b), les mesures de capacités sont effectuées à $1 \mathrm{MHz}$, la tension de polarisation inverse est de $-2 \mathrm{~V}$, la tension et la durée de remplissage sont respectivement de $2 \mathrm{~V}$ et de $1 \mathrm{~ms}$. Les courbes diffèrent par la largeur des fenêtres d'acquisitions qui varient de 1 à $5 \mathrm{~ms}$.

[DLTS spectra obtained (a) after annealing at $880{ }^{\circ} \mathrm{C}$ for $48 \mathrm{~h} \quad\left(E_{\mathrm{T}}=0,4 \mathrm{eV} ; N_{\mathrm{T}}=10^{16} \mathrm{~cm}^{-3} ; \sigma_{\mathrm{T}}=10^{-18} \mathrm{~cm}^{2}\right)$; (b) after the precedent annealing and gold diffusion at $800{ }^{\circ} \mathrm{C}$ for $3 \mathrm{~h} \quad\left(E_{\mathrm{T}}=0,38 \mathrm{eV} ; \quad N_{\mathrm{T}}=5.10^{14} \mathrm{~cm}^{-3}\right.$; $\sigma_{\mathrm{T}}=10^{-12} \mathrm{~cm}^{2}$ ). For the two spectra (a) and (b), the capacitance bridge works at $1 \mathrm{MHz}$, the reverse bias is $-2 \mathrm{~V}$, the filling pulse voltage and duration are $2 \mathrm{~V}$ and $1 \mathrm{~ms}$ respectively. The different curves correspond to emission rates or acquisition windows in the range between 1 and $5 \mathrm{~ms}$.]
Si après un recuit à $880{ }^{\circ} \mathrm{C}$ durant 30 heures, de l'or est diffusé pendant 3 heures à $800{ }^{\circ} \mathrm{C}$, la vitesse de recombinaison interfaciale $S$ prend la valeur de $8 \times 10^{3} \mathrm{~cm} . \mathrm{s}^{-1}$, valeur qu'elle atteindrait par le seul recuit d'après le tableau $I$. En revanche les longueurs de diffusion dans les grains sont deux fois plus élevées que celles mesurées après le recuit seul à $880{ }^{\circ} \mathrm{C}$ pendant $30 \mathrm{~h}$. Tout se passe, comme si l'or avait neutralisé des centres de recombinaison.

Le tableau $I$ indique que la valeur de $L_{n}$ au joint est aussi plus grande, bien que $S$ ait gardé la même valeur. Peut-être est-ce une conséquence de la trop grande taille du spot par rapport à celle du joint, qui dans la mesure de $L_{\mathrm{n}}$ intègre le voisinage immédiat des joints et aussi une partie des grains.

$\mathrm{Si}$ la diffusion d'or est effectuée après que le bicristal ait été recuit à $880{ }^{\circ} \mathrm{C}$ pendant 48 heures, des modifications importantes se produisent. Le spectre D.L.T.S. de la figure $5 b$, où l'amplitude des pics ne varie pas avec le taux d'émission, révèle l'existence d'un niveau quasi discret à $0,38 \mathrm{eV}$ (Fig. 5b), de densité $5 \times 10^{14} \mathrm{~cm}^{-3}$ et de section efficace de capture de l'ordre de $10^{-12} \mathrm{~cm}^{2}$. Par comparaison avec les effets du recuit seul, $N_{\mathrm{T}}$ a diminué, la bande de niveaux s'est resserrée et surtout $\sigma_{\mathrm{T}}$ a fortement augmenté $\left(\times 10^{6}\right)$. En revanche $S$ est sensiblement plus faible qu'après le recuit seul : $8 \times 10^{3}$ au lieu de $1,2 \times 10^{4} \mathrm{~cm} . \mathrm{s}^{-1}$. Comme dans le cas précédent la diffusion d'or restaure en partie la valeur de $L_{\mathrm{n}}$ : dans les grains comme près du joint la valeur de $L_{\mathrm{n}}$ est le triple de celle mesurée avant diffusion d'or, comme le montre la comparaison des balayages L.B.I.C. donnés par les figures $4 \mathrm{~b}$ et $4 \mathrm{~d}$.

\section{Discussion.}

Les résultats relatifs aux seuls recuits thermiques, montrent que le joint du bicristal $\Sigma=13$ brut de fabrication ne présente qu'une très faible activité électrique, ce qui est en accord avec toutes les observations antérieures.

Des recuits très longs à des températures dépassant $700{ }^{\circ} \mathrm{C}$ sont nécessaires pour modifier sensiblement l'activité recombinante de ce type de défauts. Le tableau I montre que ce n'est qu'après 30 heures à $880^{\circ} \mathrm{C}$ que des variations mesurables de la vitesse de recombinaison interfaciale sont obtenues. Toutefois, comme Ihlal [20] et Hamet [21] l'ont observé il est possible de parvenir à un résultat comparable en prolongeant un recuit à $750^{\circ} \mathrm{C}$.

Le contraste E.B.I.C. ponctué qui apparaît nettement après 48 heures à $880^{\circ} \mathrm{C}$, indique que l'activité électrique n'est pas homogène le long du joint : des groupes de centres de recombinaison se sont formés et, si la durée du recuit augmente, la largeur de la zone dénudée s'accroît. Manifestement, un diffuseur lent (ou plusieurs) est capturé par le joint. En accord 
avec les conclusions de nombreux auteurs [3-5] il est raisonnable de penser que des précipités se sont formés, dont la portée recombinante peut être observée aisément par E.B.I.C. Ces précipités ne peuvent être dus qu'à la capture d'impuretés, et éventuellement à celle des self-interstitiels provenant des grains. Après les recuits aux températures les plus élevées, une zone "dénudée " entoure le joint, comme au voisinage de la surface d'une plaquette de silicium $\mathrm{CZ}$ recuite à haute température pour déclencher le phénomène de gettering interne par les précipités de $\mathrm{SiO}_{x}$. Cette zone dénudée des plaquettes $\mathrm{CZ}$ est due à l'exodiffusion de l'oxygène par la surface libre, tandis que dans le volume, des précipités de $\mathrm{SiO}_{2}$ se sont formés. Nous pouvons imaginer qu'un mécanisme semblable se produit dans le bicristal, le joint de grains agissant comme une surface capturant l'oxygène qui quitte les grains. $\mathrm{Si}$ le joint peut aussi capturer des self-interstitiels, des précipités de $\mathrm{SiO}_{2}$ ou des agrégats de $\mathrm{SiO}_{x}$ doivent se former au joint. Les fautes d'empilement observées dans les grains peuvent également provenir de la condensation de self-interstitiels.

Le mécanisme qui vient d'être décrit se justifie par la concentration élevée en oxygène mesurée dans les bicristaux « $\mathrm{CZ}$ » $\left(10^{18} \mathrm{~cm}^{-3}\right.$ à la température ambiante) et par l'augmentation de la densité de self interstitiels lorsque des précipités de $\mathrm{SiO}_{2}$ se forment [22].

Cette participation de l'oxygène à l'activation du joint de grains a été vérifiée par les analyses de Kazmerski et al. [6] et de Pollock et al. [1]. Plus récemment, Pizzini et al. seraient parvenus à mettre en évidence la ségrégation d'oxygène aux joints de grains par Spectroscopie de masse d'ions secondaires et Spectroscopie infrarouge [5].

L'obstacle majeur vient de la valeur du coefficient de diffusion $D_{\mathrm{Oi}}$ de l'oxygène dans le silicium : à la température de $880{ }^{\circ} \mathrm{C}$ ce coefficient est de $1,4 \times 10^{-12} \mathrm{~cm}^{2} \mathrm{~s}^{-1}\left(D_{\mathrm{Oi}}=0,17 \times \exp (-2,54 / k T)\right)$ ce qui, après 48 heures, se traduit par un déplacement de $4 \mu \mathrm{m}$. Pour drainer l'oxygène des grains vers le joint sur une distance de l'ordre de $30 \mu \mathrm{m}$ (largeur de la région dénudée), il faudrait que le coefficient $D_{\mathrm{Oi}}$ soit 100 fois plus élevé. Une accélération de cette diffusivité durant les traitements thermi-

coup d'oxygène a été proposée par A. S. Oates et al. [23] et par Bergholz et al. [24] et pourrait expliquer nos résultats.

Il semble donc raisonnable d'admettre que loin du joint, des précipités de $\mathrm{SiO}_{2}$ se sont formés et participent aux recombinaisons, alors que près du joint, l'oxygène serait allé s'accumuler dans le joint (après 48 heures à $880^{\circ} \mathrm{C}$ ). Les contrastes E.B.I.C. homogènes et ponctués que nous avons obtenus sont en accord avec les travaux de Barraclough [25] qui ont montré que la nucléation des précipités d'oxygène est uniforme à $700{ }^{\circ} \mathrm{C}$ alors qu'elle se fait de préférence sur les boucles de dislocations aux environs de $900{ }^{\circ} \mathrm{C}$. C'est ce qui pourrait se produire dans les joints de grains $\Sigma=13$, au cours de nos recuits à $700{ }^{\circ} \mathrm{C}$ et à $8800^{\circ} \mathrm{C}$ [4].

L'hypothèse de la capture directe des diffuseurs rapides par le joint [7] est en contradiction avec la durée et la température élevée des recuits nécessaires à l'activation. Cette éventuelle capture, avec une accumulation au joint de tous les diffuseurs rapides contenus dans le bicristal (qui est normalement très pur) n'explique pas l'apparition de la zone dénudée autour du joint de la figure 3. Dans ces zones, les longueurs de diffusion sont plus élevées que dans le reste des grains, où elles s'effondrent. Si le joint avait capturé tous les diffuseurs rapides présents dans le cristal, les longueurs de diffusion devraient avoir la même valeur élevée dans l'intégralité des grains, ce qui n'est pas le cas d'après les résultats contenus dans le tableau $I$ et les figures 4.

L'insensibilité du joint et des grains à la présence d'atomes d'or interstitiels introduits dans le bicristal après des recuits à $700{ }^{\circ} \mathrm{C}$ pendant 2 heures et à $800{ }^{\circ} \mathrm{C}$ pendant 3 heures, montre que la capture directe de ces atomes par le joint est soit peu probable, soit sans effet électrique. La deuxième éventualité nous paraît plus vraisemblable, compte tenu de l'aptitude de l'or à se fixer sur les défauts et les surfaces.

Lorsque l'or diffuse dans un bicristal préalablement recuit beaucoup plus énergétiquement, alors un effet se produit dans les joints comme dans les grains, mais c'est une passivation et non une augmentation de l'activité recombinante des joints et des défauts intragrains qui est observée.

Même si le mécanisme de la passivation par l'or n'est pas actuellement compris, il est raisonnable de penser qu'elle ne peut se réaliser qu'après capture des atomes métalliques par les précipités qui se seraient formés au joint et dans les grains. Il est alors normal que des microanalyses révèlent la présence d'atomes métalliques dans les précipités [7]. Les résultats de Pollock et al. [1] vont dans ce sens. Bien qu'ils aient été obtenus avec des polycristaux, ces résultats montrent que l'oxygène est bien capturé par les joints où il forme des précipités, et lorsque du

tal, les atomes de cuivre et d'oxygène sont ségrégés aux mêmes endroits.

Dans ce qui précède, nous n'avons pris en compte que les valeurs des coefficients de diffusion comme critère de discussion, et nous n'avons pas considéré le ou les mécanismes de capture des impuretés par le fait que près des joints de grains et des précipités il peut toujours y avoir des boucles de dislocations, voire des fautes d'empilement ou des régions soumises à des contraintes. La capture d'impuretés métalliques ne devrait alors pas poser de problème même à 
température modérée et il doit en être de même pour l'oxygène.

Nous continuons donc à penser que l'oxygène dissous dans le bicristal est, après ségrégation par le joint de grains, le principal responsable de l'augmentation de l'activité recombinante du joint $\Sigma=13$. Une fois que des précipités d'oxyde se sont formés, il est tout à fait possible que des impuretés métalliques soient capturées et que suivant leur concentration initiale dans le cristal, une neutralisation partielle des centres de recombinaison puisse se produire.

Nous reconnaissons que nous sommes restés dans le domaine des hypothèses et que nous n'avons pas, pour l'instant, réussi à mettre en évidence de façon indiscutable la présence de précipités $\mathrm{SiO}_{2}$ ou de complexes $\mathrm{SiO}_{x}$ aux joints des cristaux. Mais d'autres auteurs l'ont fait avait nous.

\section{Conclusion.}

L'activité recombinante des joints du type $\Sigma=13$ dans des bicristaux obtenus par tirage « $\mathrm{CZ}$ » est initialement faible et dépend très fortement des traitements thermiques appliqués à l'échantillon. Cette activité ne prend des valeurs importantes qu'après des recuits prolongés sous gaz neutre à des températures supérieures à $700{ }^{\circ} \mathrm{C}$. Il semble raisonnable d'admettre que l'oxygène dissous dans le bicristal participe à l'activation du joint après ségrégation par le défaut. Cette participation serait même prépondérante. En effet l'introduction volontaire dans le cristal d'une impureté métallique, connue pour son aptitude à diffuser rapidement et à former des centres de recombinaison, n'accroît pas l'activité recombinante du joint, et même la réduit, tout en restaurant les longueurs de diffusion des porteurs minoritaires.

\section{Bibliographie}

[1] Pollock G. A., Deline V. R. et Furman B. K., Grain boundaries in semiconductors Ed. H. J. Leamy, G. E. Pike and C. H. Seager, $M R S$ Symp. Proc. (North Holland New York) 5 (1982) 71.

[2] Kazmerski L. L., Proc. of 5th E. C. Photovoltaic Solar Energy Conf. (D. Reidel Pub. Comp.) 1983, p. 40.

[3] Battistella F., Rocher A. et George A., Oxygen, Carbon, Hydrogen and Nitrogen in Crystalline Silicon Ed. J. C. Mikkelsen Jr., S. J. Pearton, J. W. Corbett and S. J. Pennycook, MRS Symp. Proc. (North Holland, New York) 59 (1986) 347.

[4] Sutzler F. J. et Queisser H. J., J. Appl. Phys. 60 (1986) 3910.

[5] Pizzini S., Cagnoni P., Sandrinelli A., Anderle M. et Canteri R., Appl. Phys. Lett. 51 (1987) 676.

[6] Kazmerski L. L., Polysilicon Films and Interfaces, Eds. C. Y. Wong, C. V. Thompson and K. N. Tu, MRS Symp. Proc. (North Holland New York) 106 (1988) 199.

[7] Aucouturier M., Broniatowski A., Chari A. et MAURICE J. L., Polycrystalline semiconductors Eds. J. H. Werner, H. S. Moller et H. P. Strunk (Springer Verlag Berlin) 35 (1989) p. 64.

[8] Schmalz K., KiUcht F. G., Kose H., Richter H. et Tittelbach-Helmrich K., Phys. Status Solidi (a) 100 (1987) 567.

[9]Bronner G. B. et Plummer J. D., J. Appl. Phys. 61 (1987) 5286.

[10] Aubert J. J. et Bacmann J. J., Revue de Phys. Appl. 22 (1987) 515.
[11] Battistella F., Thèse de Docteur Ingénieur, Université de Toulouse (1985).

[12] Bullis W. M., Solid State Electron. 9 (1966) 143.

[13] Stolwijk N. A., Holzl J., Frank W., Weber E. R., Mehrer H., Appl. Phys. A 39 (1986) 37.

[14] Lang D. V., J. Appl. Phys. 45 (1974) 3023.

[15] Spencer M., Stall R., Eastman L. F. et Wood C. E. C., J. Appl. Phys. 50 (1979) 8006.

[16] Cheng L. J. et Shyu C. M., Semiconductor Silicon 1981, Eds. H. R. Huff, R. J. Kriegler et Y. Takeishi (The Electrochem. Soc. Inc. ; Pennington) 81-5 (1981) 390.

[17] Pasquinelli M., Thèse de Doctorat en Sciences, Université de Marseille (1988).

[18] MARTINUzzi S., dans référence [7] p. 148.

[19] CARd H. C. et Yang E. S., IEEE Trans. Elec. Dev. ED 24 (1977) 397.

[20] Ilhal A., Nouet G., dans reference [7] p. 77.

[21] HAMET H., Thèse de Doctorat en sciences, Université de Caen (1989).

[22] Forbes L., Whitver F. D. et Peng J. D., dans Impurity Diffusion and Gettering in Silicon, Ed. R. B. Fair, MRS Symp. Proc. (New York) 36 (1985) 257.

[23] Oates A. S. et Newman R. C., Appl. Phys. Lett. 49 (1986) 262.

[24] Bergholz W., Hutchinson J. C. et Pirouz P., $J$. Appl. Phys. 58 (1985) 3419.

[25] Barraclough K. G., dans Aggregation phenomena of point defects in silicon, Eds. E. Sirtl et J. Gorrissen (The Electrochem. Soc. New Jersey) 1983, p. 176. 